\title{
On the correlation between residual stresses and displacements of the polycrystalline lattice: an experimental strategy
}

\author{
${ }^{1}$ F.V. Díaz, ${ }^{1}$ F.N. Rosso, ${ }^{1}$ A.C. Walker, and ${ }^{2}$ A.P.M. Guidobono \\ ${ }^{1}$ Departamento de Ingeniería Electromecánica, Universidad Tecnológica Nacional (FRRa), \\ Acuña 49, 2300 Rafaela \\ ${ }^{2}$ División Metrología Dimensional, Instituto Nacional de Tecnología Industrial, \\ Ocampo y Esmeralda, 2000 Rosario \\ Argentina
}

Received: October 12, 2020. Revised: November 20, 2020. Accepted: November 23, 2020. Published: November 24, 2020.

\begin{abstract}
This work aims to propose a micro-indent method to evaluate the correlation between the relaxation of residual stresses and the shape changes of the polycrystalline lattice in specimens of a rolled plate of $\mathrm{AA}$ 6082-T6 aluminium alloy. Rolling strengthens the surface of the plate by introducing compressive residual stresses. Then, these stresses are relaxed by thermal distension. The method of micro-indents allowed measuring residual displacements with an error below $\pm 300 \mathrm{~nm}$. The results obtained reveal that the rolling direction plays a vital role in terms of asymmetric expansion of the lattice. Furthermore, the lattice accumulates and restores elastic strain energy in the clockwise and anti-clockwise direction of rotation, alternatively. Finally, when the stress release process is finishing, the lattice adjusts the angle of rotation to approximate to the initial geometric shape.
\end{abstract}

Keywords - Elastic strain energy, method of microindents, residual stresses, rolling direction.

\section{INTRODUCTION}

$\mathrm{R}$ ESIDUAL stresses in components of structures or machines can cause catastrophic failures [1]. These stresses are present in a solid, even if there are no externally applied forces and moments. Different processing or joining operations such as welding, machining, extrusion, forging or rolling can introduce tensile or compressive residual stresses, which generate opposite effects. Tensile stresses increase risks of failure in components subjected to fatigue, corrosion, or wear [2]. On the other hand, compressive stresses are deliberately induced by different methods to strengthen the surface of a component [3].

When a rolled plate of aluminium alloy is acquired for structural purposes, the normal and tangential components of residual stress [4] introduced during manufacture are unknown. Determining the sign and levels of these stresses is critical. For evaluating risks, the normal components of residual and service stress must be added. If the sum is unknown and its real value approaches or exceeds the maximum allowable stress, the collapse would occur at the beginning of its useful life.

Several techniques are applied for residual stress determination. These techniques can be classified as direct and indirect [5]. In the first, the stress values are obtained from the measurement of physical properties modified by residual stress introduction. In the second, the equilibrium of forces and/or moments is broken by cutting or the removal of any part of the solid. Then, the residual displacements are measured. X-ray diffraction [6], [7] and hole-drilling method [8], [9] are the most used direct and indirect techniques, respectively.

Furthermore, in the last two decades, different methods of micro and nano-indentation have been developed and optimized for measuring residual stresses [10], [11]. Wyatt and Berry [12], [13] proposed a technique based on displacements of several micro-indents. The displacements occur when the residual stress field is relaxed from thermal distension. Then, this technique was improved by integrating a universal measuring machine, which is a multipurpose, robust and highly accurate measurement tool [14]. Afterwards, this optimized technique allowed determining, with high accuracy, residual stress fields introduced from milling operations [15]-[17]. 
The purpose of this work is to demonstrate the sensitivity and feasibility of a micro-indent approach to explore the correlation between the residual stress relaxation in samples of a rolled plate of AA 6082-T6 aluminium alloy and the corresponding changes of shape of the polycrystalline lattice. From the link between the microstructure (changes in the shape of the lattice) and behaviour (relaxation of residual stresses), this work evaluates the changes in the mechanical properties (compressive residual stresses) of the material assessed. The residual stresses were relieved by thermal distension and evaluated during $80 \mathrm{~min}$. It is worth noting that for the case of the present alloy, the study of the link between residual stresses and the corresponding changes in the polycrystalline lattice does not show any antecedent in the literature. In the present work, these changes are evaluated from eight linear micro-indents [14]. The tangential and normal components of residual stress were determined in the centroids of five geometric figures by using a model of plane stress [4]. The results obtained reveal the strong influence of the rolling direction on the normal components relieved, and besides, on the expansion generated in the lattice. Furthermore, the results demonstrate that the tangential component induces to the lattice to work as a rotational spring, accumulating and restoring elastic deformation energy in the clockwise and anti-clockwise direction of rotation, alternatively. Finally, a good correlation was obtained between all components of residual stress and the expansion, distortion, and rotation generated in the polycrystalline lattice of the alloy evaluated.

\section{MATERIALS AND METHODS}

\section{A. Micro-indent Method}

As above-mentioned, a method of micro-indents was used to evaluate the release of different components of residual stress, and also, the displacements associated with the polycrystalline lattice. Several samples of AA 6082-T6 aluminium alloy were taken from a hot rolled plate $4 \mathrm{~mm}$ thick. It is worth noting that this material is widely used in structural applications in the maritime and shipping industries as well as for precision parts in the automotive industry.

In this work, the residual stress values were determined in the centroids of a square and four triangles, which are fractions of the square. Fig. 1 shows a sample evaluated; the vertices of the square are I, II, III and IV, and the vertices of the four triangles are: (I, II, III), (II, III, IV), (I, II, IV) and (I, III, IV). The points 1,2, 4 and 5 are the centroids of the triangles, and point 3 is the centroid of the square. It is worth noting that the $x$-axis corresponds to the rolling direction. Each vertex is the intersection of two linear micro-indents, which are introduced using a special indenter device, which is located at the main header of the universal measuring machine (GSIP MU-314), as shown in Fig. 2. Therefore, each linear indent is introduced and measured from the measuring machine used in this work. It is worth noting that this machine performs measurements in three rectangular coordinate axes, whose displacements are
400, 100 and $128 \mathrm{~mm}$ for the $x, y$ and $z$ axes, respectively, with an optical magnification of $129 \mathrm{X}$ and a smallest reading of 100 nm.
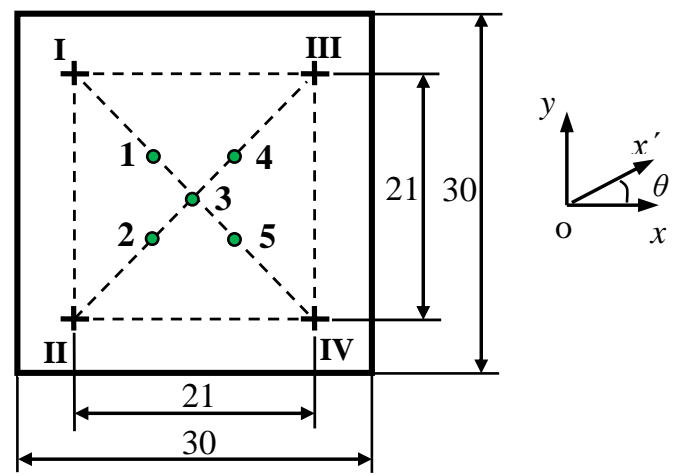

Fig. 1. Distribution of micro-indents in a sample evaluated. The units are $\mathrm{mm}$ and the thickness is $4 \mathrm{~mm}$.

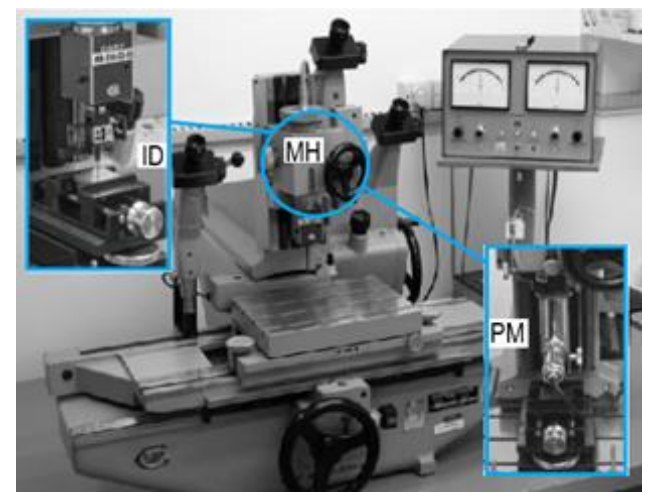

Fig. 2. Universal measuring machine $(\mathrm{MH}$, main header of the machine; ID, indenter device; PM, precision microscope)

The following procedure of measurement was performed for each sample. First, the linear micro-indents are introduced using the indenter device. Then, the coordinates of the microindents are optically measured by using a precision microscope, which is also mounted in the main header of the measuring machine. Afterwards, the residual stresses are relieved in an oven (Dalvo HM2). Finally, the sample is repositioned and optically aligned, and the coordinates of the micro-indents are again measured. The thermal distensions, corresponding to the third phase of this procedure, were carried out at $300{ }^{\circ} \mathrm{C}$ for 20,60 and $80 \mathrm{~min}$.

Fig. 2 shows the high precision microscope used in this work. From this microscope, which is an essential accessory, the coordinates of the micro-indents were measured with accuracy better than $\pm 100 \mathrm{~nm}$ [14]. It is worth noting that this accessory provides the essential functions of referencing and magnification [18], and is commonly used to measure dimensions and shapes of different mechanical components.

\section{B. Residual Stress Determination}

From the coordinates of each linear micro-indent before and after the thermal distension, the residual displacements ( $u$ and 
$v)$ and strain components $\left(\varepsilon_{x}, \varepsilon_{y}\right.$ and $\left.\gamma_{x y}\right)$ are calculated, in each centroid evaluated, following a procedure developed in [14]. Then, assuming that the material evaluated is under plane stress conditions [4], the normal and tangential components of residual stress were obtained using

$$
\begin{gathered}
\sigma_{x^{\prime}}=\frac{\sigma_{x}+\sigma_{y}}{2}+\frac{\sigma_{x}-\sigma_{y}}{2} \cos 2 \theta+\tau_{x y} \cdot \sin 2 \theta \\
\tau_{x^{\prime} y^{\prime}}=-\frac{\sigma_{x}-\sigma_{y}}{2} \sin 2 \theta+\tau_{x y} \cdot \cos 2 \theta
\end{gathered}
$$

where $\sigma_{x}, \sigma_{y}$ and $\tau_{x y}$, obtained from $\varepsilon_{x}, \varepsilon_{y}$ and $\gamma_{x y}$, are the residual stress components in the sample coordinates system [4], and $\theta$ is the angle corresponding to the direction of evaluation (see Fig. 1).

Although the residual stress components were obtained from samples treated at $300{ }^{\circ} \mathrm{C}$ for 20,60 and $80 \mathrm{~min}$, effects of plasticity and creep strains are considered negligible because a weak macro-stress field is evaluated. Also, the holding times are moderate [19], [20]. It is worth noting that for the temperature of $300{ }^{\circ} \mathrm{C}$ and very low residual stresses, the dominant strain mechanism is bulk diffusion through the lattice (Nabarro-Herring creep) [21], [22]. Furthermore, the thermal stresses are also considered negligible due to the geometry of the sample is very simple, the thickness is small, and the cooling rate is slow [23].

As above-mentioned, the measurement error of the residual displacements is $\pm 300 \mathrm{~nm}$. During each measurement of micro-indent, the control of the room temperature is very critical. In the present experimental procedure, the coordinates of each micro-indent were measured with a variation of temperature within $20 \pm 0.2{ }^{\circ} \mathrm{C}$ and a rate of fewer than 0.01 ${ }^{\circ} \mathrm{C} / \mathrm{min}$. It should be noted that the error of measurement will significantly increase if the rate is higher than the value expressed.

\section{RESULTS AND DISCUSSION}

\section{A. Residual Stress Relaxation}

Fig. 3(a) shows the stress relaxation corresponding to the component $\sigma_{x}$, in each centroid evaluated. This stress component is compressive. It is worth noting that the direction associated with $\sigma_{x}$ corresponds to the rolling direction. As expected, the maximum distension occurs from 0 to $20 \mathrm{~min}$ (phase 1). Then, from 20 to $60 \mathrm{~min}$ (phase 2), the gradient is lower. Furthermore, in phases 1 and 2, the evolution is similar in all points. Afterwards, from 60 to $80 \mathrm{~min}$ (phase 3), the stress release is slightly different at centroids 1 and 5 .

On the other hand, Fig. 3(b) shows the relaxation of the component $\sigma_{y}$, whose direction is perpendicular to the rolling direction. The evolution of $\sigma_{x}$ and $\sigma_{y}$ is similar, although the values of $\sigma_{y}$ are lower. In phases 1 and 2, the gradients of $\sigma_{x}$ are higher than those of $\sigma_{y}$, but, in phase 3 , the gradients are similar. Moreover, the component $\sigma_{y}$ shows different changes in phase 2. In points 1 and 2 (left zone of the sample), the stress release is slightly higher than in points 3 (central zone), 4 and 5 (right zone).

The relaxation of the tangential component $\tau_{x y}$ is shown in Fig. 4. The values obtained are much smaller than those of $\sigma_{x}$ and $\sigma_{y}$. The difference is approximately an order of magnitude. Note that all values of $\tau_{x y}$ are within a minimal range (7 MPa). Although the values obtained are low, the evolution shows changes of sign in the gradients of consecutive phases. In phase 1, the gradients are similar for all points. Points 1 and 4, aligned in the rolling direction, have similar gradient values in phase 2. The same occurs for points 2 and 5, which are also aligned in the rolling direction.
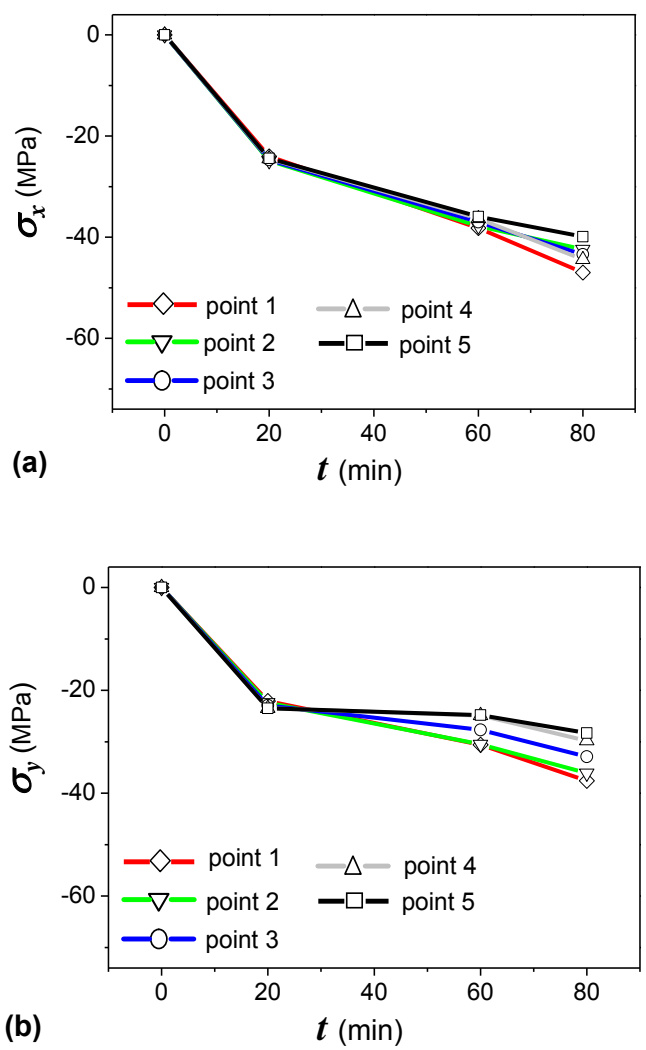

Fig. 3. Relaxation of the stress components (a) $\sigma_{x}$ and (b) $\sigma_{y}$ in all centroids evaluated.

The components $\sigma_{x}, \sigma_{p}, \sigma_{y}$ and $\sigma_{q}$, corresponding to the centroid 3, are shown in Fig. 5. $\sigma_{p}$ and $\sigma_{q}$ are called principal components of residual stress, and the directions associated are called principal directions [24]. It worth noting that $\sigma_{p}$ and $\sigma_{q}$ correspond to the maximum and minimum value of $\sigma_{x}$, respectively (see (1)). A notable feature is that the principal components $\sigma_{p}$ and $\sigma_{q}$ behave like $\sigma_{x}$ and $\sigma_{y}$, respectively. Therefore, for the centroid evaluated, the principal directions of residual stress are very close to the coordinate axes ( $x$ and $y$ ). Consequently, the rolling direction is very near the principal direction associated with the maximum component $\sigma_{p}$. 


\section{B. Displacements of Vertices}

The displacements generated by thermal distensions were used to evaluate the lattice shape changes. These displacements were evaluated at the vertices (I, II, III and IV) of the square (see Fig. 1). As above-mentioned, each vertex corresponds to the intersection of two linear micro-indents. Fig. 6 shows the displacements associated with each phase. In phase 1 , each vertex moves to point $A$. Then, the segments $A B$ and $\mathrm{BC}$ correspond to phases 2 and 3 , respectively.

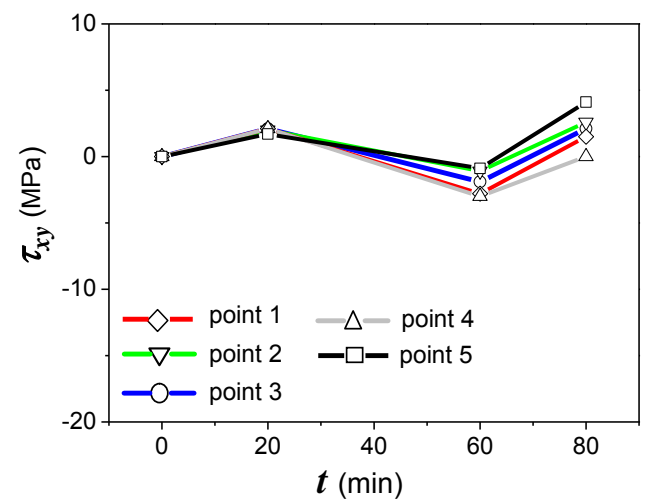

Fig. 4. Relaxation of the stress component $\tau_{x y}$ in all centroids evaluated.

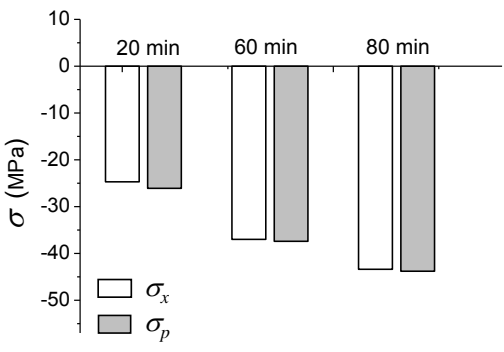

(a)

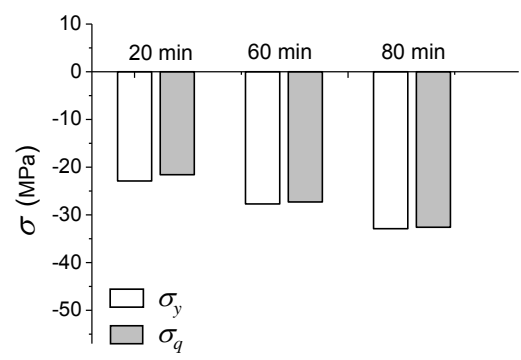

(b)

Fig. 5. Relaxation of the stress components (a) $\sigma_{x}$ and $\sigma_{p}$, (b) $\sigma_{y}$ and $\sigma_{q}$ in the centroid 3 .

All displacements are well organized in phase 1. The microindents move following a small angle regarding a local coordinate axis. Then, in phase 2 , the displacements change of direction. All micro-indents moves towards the other coordinate axis (point B). Afterwards, a new change of direction occurs in phase 3 but following a small angle. The micro-indents are bringing near the bisector of the local coordinate system (point $\mathrm{C}$ ). It should be noted that at each vertex, points $\mathrm{A}$ and $\mathrm{B}$ are close to the local coordinate axes. Therefore, the absolute displacement is quasi-directional in phases 1 and 2. Instead, the point $\mathrm{C}$ is close to the bisector for each vertex, which indicates a more balanced absolute displacement in both directions ( $\mathrm{x}$ and $\mathrm{y}$ ) for phase 3 .

Because the displacements shown in Fig. 6 were amplified for clarity, Table I shows the values measured in each segment. As expected, the maximum displacements correspond to phases 1 and 2. On the other hand, the smallest displacements occur in phase 3 . In this phase, the more significant displacements, which occur in opposite vertices, are quasi-parallel to the $x$-axis (rolling direction).

Fig. 6 and Table 1 show vertices I and IV have similar displacement geometry and values. The same occurs for vertices II and III. Therefore, diagonals I-IV and II-III are axes of symmetry in terms of displacement geometry and values.

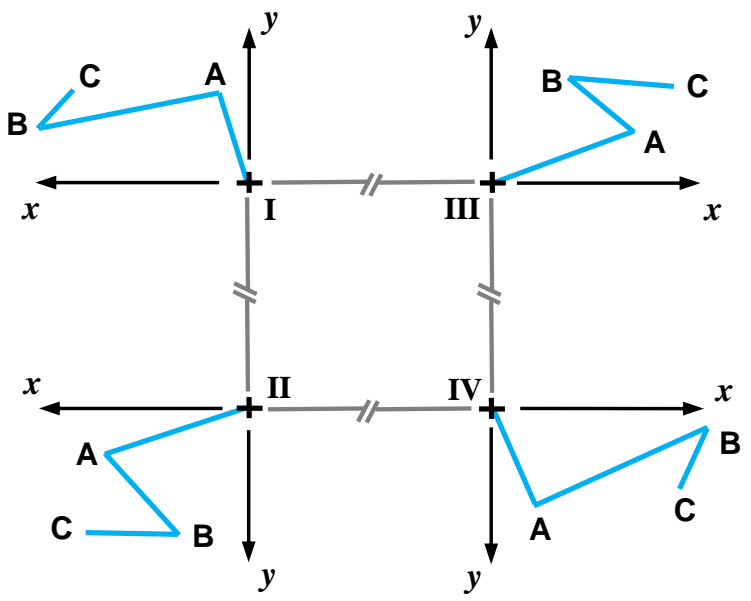

Fig. 6. Displacements of the vertices I, II, III and IV. A, B and C are the displacement finals of the phases 1,2 and 3, respectively.

Table I. Displacements in each vertex

\begin{tabular}{|c|c|c|c|}
\hline \multicolumn{4}{|c|}{ Displacement $(\mu \mathrm{m})$} \\
\hline Vertex & Phase 1 & Phase 2 & Phase 3 \\
\hline I & 2.9 & 5.4 & 1.5 \\
\hline II & 4.4 & 3.2 & 2.8 \\
\hline III & 4.3 & 2.5 & 3.1 \\
\hline IV & 3.3 & 5.6 & 2.0 \\
\hline
\end{tabular}

\section{Shape Changes of the Polycrystalline Lattice}

Fig. 7 shows the expansion, distortion and rotation of the original shape, which occur when the components $\sigma_{x}, \sigma_{y}$ and $\tau_{x y}$ are released. In phase 1 , the expansion, which is slightly asymmetric, is due to the normal components relieved are compressive. On the other hand, the clockwise rotation would be caused by distortion, which occurs when the tangential component $\tau_{x y}$ is relieved. The asymmetrical character of the expansion is more notorious in phase 2. Furthermore, the 
rotation of the lattice is anti-clockwise, and the distortion is more significant than in the previous phase. The release of the component $\tau_{x y}$, in phase 3 , induces to a geometrical shape harmonizing with the initial form because three sides are parallel to three sides of the initial square. Moreover, the expansion generated by the components $\sigma_{x}$ and $\sigma_{y}$ is small, and therefore, the figure remains elongated in the direction $x$ (rolling direction).

(a)

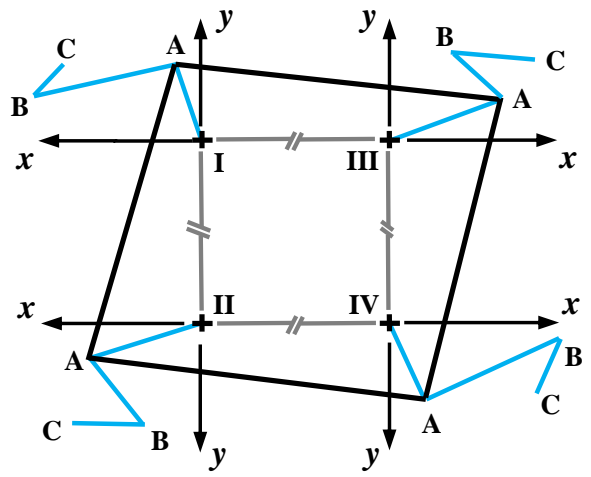

(b)

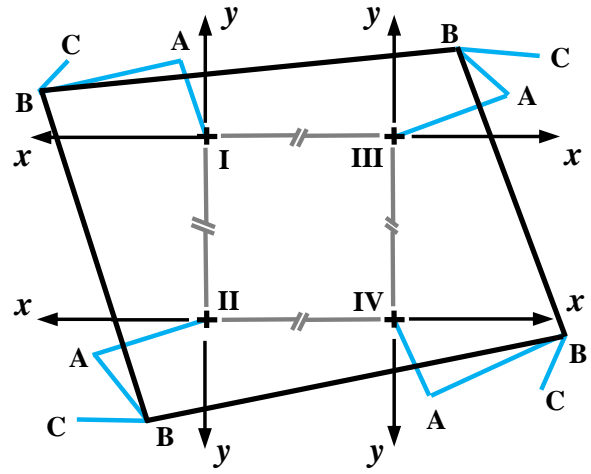

(c)

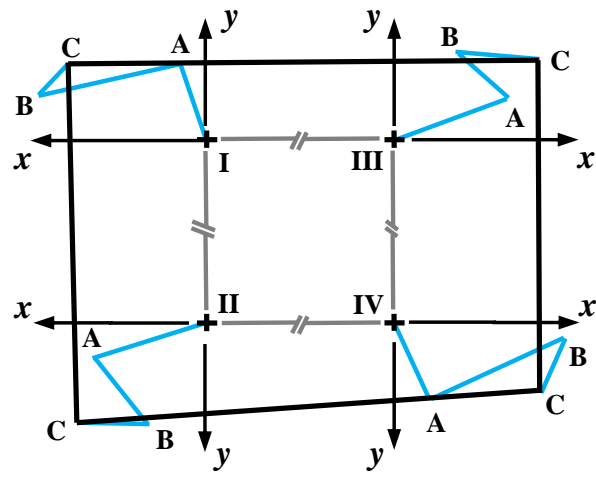

Fig. 7. Shape changes corresponding to the phases (a) 1, (b) 2 and (c) 3 .

The results obtained show that the polycrystalline lattice goes through different phases to minimize its elastic strain energy [25]. At the beginning of the stress release process (phase 1), the lattice undergoes expansion, distortion and rotation. The original square is transformed in a parallelogram rotated. However, this parallelogram is symmetric and harmonic because each component relieved $\left(\sigma_{x}, \sigma_{y}\right.$ and $\left.\tau_{x y}\right)$ shows similar values in all points, as shown in Figs. 3 and 4. The expansion is asymmetric due to the rolling process carried out in the alloy evaluated, which was performed in the $x$ direction, and therefore $\sigma_{x}>\sigma_{y}$, as shown in Fig. 3. The release of the components $\sigma_{x}$ and $\sigma_{y}$ continues in phase 2, and consequently, the lattice continues to expand. The parallelogram generated in this phase is slightly irregular since $\sigma_{y}$, and $\tau_{x y}$ have gradients somewhat different in each centroid (see Figs. 3 and 4). In phase 3, the weak release of $\sigma_{x}$ and $\sigma_{y}$ correlates very well with the expansion reduced of the lattice regarding the previous phase. Moreover, the shape generated in this phase correlates well with the components relieved because $\sigma_{x}, \sigma_{y}$ and $\tau_{x y}$ show similar gradients in the different centroids (see Figs. 3 and 4).

On the other hand, the rotation of the lattice would be activated from distortion, which is generated by the release of the component $\tau_{x y}$. In Fig. 8, a model of distortion causing the lattice rotation is proposed. In phase 1 , the component $\tau_{x y}$ is relieved in the clockwise direction, and therefore, the distortion generates rotation in the clockwise direction. At the beginning of phase 2, the lattice is stressed in the clockwise direction; therefore, the rotation will be in the anti-clockwise direction. Finally, at the beginning of phase 3, the lattice is stressed in the anti-clockwise direction, which will generate the clockwise rotation. This movement of rotation, oscillating between the clockwise and anti-clockwise directions, correlates very well with the changes of sign of the component $\tau_{x y}$, which are shown in Fig. 4
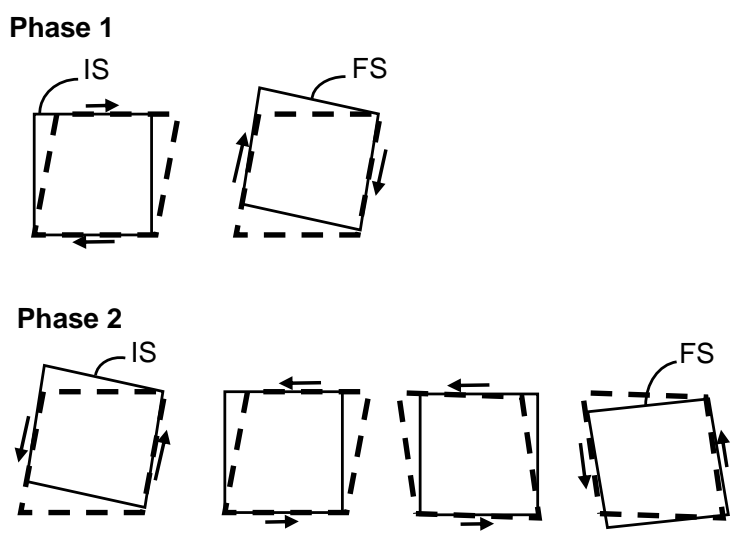

Phase 3

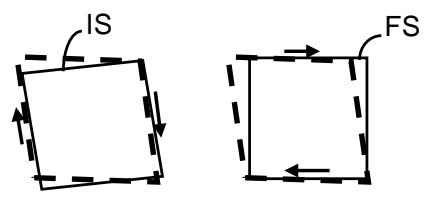

Fig. 8. Model of distortion associated with the relaxation of $\tau_{x y}$ (IS, initial shape of the phase; FS, final shape of the phase).

For obtaining a state of low elastic strain energy [26], the relaxation of the component $\tau_{x y}$ induces to the lattice to work as a rotational spring, accumulating and restoring elastic strain energy in the clockwise and anti-clockwise directions, 
alternatively. Finally, during phase 3 , the same component $\tau_{x y}$ would induce to the lattice to retrieve the initial geometrical shape (square). Therefore, the rotation angle would adjust for such purpose.

\section{CONCLUSION}

This work proposes a micro-indent method to explore the correlation between the thermal relaxation of different components of residual stress and the corresponding changes of shape undergoing the polycrystalline lattice. The evaluation was carried out from samples of a rolled plate of AA 6082-T6 aluminium alloy. The main findings are:

-The proposed method has proven to be very efficient and accurate to determine residual displacements.

-The normal components of residual stress introduced by rolling are compressive (beneficial) and are preferably relieved along the rolling direction.

-Although the tangential component introduced is an order of magnitude smaller than the normal components, it has a relevant role in terms of changes in the polycrystalline lattice.

-At the barycenter of all samples evaluated, the principal direction of the residual stress is very near the rolling direction.

-An expansion of the lattice accompanies the relief of the normal components. On the other hand, the release of the tangential component generates distortion and rotation in the lattice.

-The rotation of the lattice occurs in the clockwise and anticlockwise directions, alternatively. Therefore, the lattice would be accumulating and restoring elastic strain energy in the rotational direction.

-At the end of the distension process, the lattice seeks to develop a geometric shape, which is also governed by the tangential stress component relieved.

\section{ACKNOWLEDGMENT}

The authors acknowledge the financial support of Universidad Tecnológica Nacional and Consejo Nacional de Investigaciones Científicas y Técnicas of Argentina.

\section{References}

[1] M.N. James, "Residual stress influences on structural reliability," Eng. Fail. Analysis, vol. 18, no. 8, pp. 19091920, 2011.

[2] P.J. Withers, "Residual stress and its role in failure," Rep. Prog. Phys., vol. 70, pp. 2211-2264, 2007.

[3] M. Kobayashi, T. Matsui and Y. Murakami, "Mechanism of creation of compressive residual stress by shot peening," Int. J. Fatigue, vol. 20, pp. 351-357, 1998.

[4] B. J. Goodno and J. M. Gere, Mechanics of Materials. $9^{\text {th }}$ Edition. New York: Cengage Learning, 2017.
[5] J. Guo, H. Fu, B. Pan and R. Kang, "Recent progress of residual stress measurement methods: A review," Chinese J. Aer., to be published (Corrected proof 2019).

[6] I.C. Noyan and J.B. Cohen, Residual Stress Measurement by Diffraction and Interpretation. Berlin: Springer-Verlag, 1987.

[7] M. Matsuda, K. Okita, T. Nakagawa and T. Sasaki, "Application of X-ray stress measurement for residual stress analysis by inherent strain method -Comparison of $\cos \alpha$ and $\sin 2 \psi$ method-," Mech. Eng. J., vol. 4, no. 5, pp. 1-13, 2017.

[8] N.J. Rendler and I. Vigness, "Hole-drilling strain-gage method of measuring residual stresses," Exp. Mech., vol. 6, pp. 577-586, 1966.

[9] F.V. Díaz, G.H. Kaufmann and O. Möller, "Residualstress determination using blind-hole drilling and digital speckle pattern interferometry with automated data processing, " Exp. Mech., vol. 41, pp. 319-323, 2001.

[10] S. Suresh and A. Giannakopoulos, "A new method for estimating residual stresses by instrumented sharp indentation," Acta Mater., vol. 46, pp. 5755-5767, 1998.

[11] G. Peng, Z. Lu, Y. Ma, Y. Feng, Y. Huan and T. Zhang, "Spherical indentation method for estimating equibiaxial residual stress and elastic-plastic properties of metals simultaneously,” J. Mater. Res., vol. 33, 884-897, 2018.

[12] J.E. Wyatt and J.T. Berry, "A new technique for the determination of superficial residual stresses associated with machining and other manufacturing processes," J. Mater. Proc. Tech., vol. 171, pp. 132-140, 2006.

[13]J.E. Wyatt, J.T. Berry and A.R. Williams, "Residual stresses in aluminum castings," J. Mater. Proc. Tech. vol. 191, no. 1-3, pp. 170-173, August 2007.

[14]F.V. Díaz, R.E. Bolmaro, A.P.M. Guidobono and E.F. Girini, "Determination of residual stresses in high speed milled aluminium alloys using a method of indent pairs," Exp. Mech., vol. 50, no. 2, pp. 205-215, February 2010.

[15]F.V. Díaz and C.A. Mammana, "Study of residual stresses in conventional and high-speed milling," in Milling: Operations, Applications and Industrial Effects, L.A. Filipovic, Ed. New York: Nova Science Publishers, Inc., 2012, pp. 127-155.

[16]F.V. Díaz, C.A. Mammana and A.P.M. Guidobono, "Evaluation of residual stresses in low, medium and high speed milling," Res. J. App. Sci. Eng. Tech., vol. 11, pp. 252-258, 2015.

[17] S. Vottero, F.V. Díaz, C.A. Mammana and A.P.M. Guidobono, "Analysis of residual stresses in high speed milled aluminum alloys," Res. J. App. Sci. Eng. Tech., vol. 4, pp. 119-123, 2017.

[18] C. L. Dotson, Fundamentals of Dimensional Metrology. New York: Cengage Learning, 2015.

[19] W. De Bruin, "Dimensional stability of materials for metrological and structural applications," Ann. CIRP, vol. 31, pp. 553-560, 1982.

[20] R. Seifi and D. Salimi-Majd, "Effects of plasticity on residual stresses measurement by hole drilling method," Mech. Mater., vol. 53, pp. 72-79, 2012. 
[21] G. Schoeck, "Theories of creep," in Mechanical Behavior of Materials at Elevated Temperatures, J.E. Dorn, Ed. New York: McGraw-Hill Book Company Inc., 1961, pp: 79-107.

[22] M.F. Ashby "A first report on deformation-mechanism maps," Acta Metall., vol. 20, pp. 887-897, 1972.

[23] N. Noda, R.B. Hetnarski and Y. Tanigawa. Thermal Stresses. New York: Taylor \& Francis, 2003.

[24]F. P. Beer, E. Russell Johnston Jr., J. T. DeWolf and D. F. Mazurek, Mechanics of Materials, $8^{\text {th }}$ Edition. New York: McGraw-Hill Education, 2019.

[25] W. Ding, Y. Liu, J. Xie, L. Sun, T. Liu, F. Yuan, and J. Pan, "Effect of carbide precipitation on the evolution of residual stress during tempering," Metals, vol. 9, pp. 1-14, 2019.

[26] J. Yang, H. Jiang, Z. Yao, J. Dong, "Limitations of calculating stress relaxation limit by function-fitting of Inconel718 superalloy," Mater. Letters, vol. 221, pp. 8992, 2018.

\section{Contribution of individual authors to the creation of a scientific article}

F.V. Díaz: Design of experiments, conducting research, analysis and writing of the manuscript.

F. N. Rosso: Implementation of research, analysis and evaluation of results.

A. C. Walker: Implementation of research, analysis and evaluation of results.

A. P. M. Guidobono: Measurement design, analysis and evaluation of results.

\section{Creative Commons Attribution License $\mathbf{4 . 0}$ (Attribution 4.0 International, CC BY 4.0)}

This article is published under the terms of the Creative Commons Attribution License 4.0

https://creativecommons.org/licenses/by/4.0/deed.en_US 